\title{
Polypoidal Choroidal Vasculopathy
}

\author{
Sana Nadeem \\ Department of Ophthalmology, Foundation University Medical College \& Fauji Foundation Hospital, Rawalpindi
}

\begin{abstract}
A 76-year-old, hypertensive lady, presented with a three year history of gradual decrease in vision in her right eye. Examination revealed a large, bullous, serous pigment epithelial detachment (PED) of right fovea, a choroidal neovascular membrane, clusters of hard exudates, drusen and surrounding, multifocal, small PEDs. The left eye showed a series of small PEDs mostly on the inferior macula, pigmentary disturbance of the retinal pigment epithelium and scant hard exudates. A diagnosis of Polypoidal choroidal vasculopathy was made. We decided to treat her with intravitreal Bevacizumab injections in her right eye. At 18 months of follow up, her PEDs had resolved and visual acuity had improved from 6/60 OD to 6/36.
\end{abstract}

Key Words: Polypoidal Choroidal Vasculopathy, Age Related Macular Degeneration, Pigment Epithelial Detachment, Choroidal Neovascular Membrane.

How to Cite this Article: Nadeem S. Polypoidal Choroidal Vasculopathy. Pak J Ophthalmol. 2021, 37 (2): 239242.

Doi: http://doi.org/10.36351/pjo.v37i2.972

\section{INTRODUCTION}

The term Polypoidal choroidal vasculopathy (PCV) was first coined by Yannuzzi in 1982, as an idiopathic choroidal vascular disease. In 1984, Kleiner termed it 'posterior uveal bleeding syndrome'. It is characterized by a bilateral multiple recurrent serosanguinous pigment epithelial detachments (PEDs) and orange-red aneurysmal dilatations called polyps. It is believed by many to be a subtype of neovascular age related macular degeneration with an abnormal branching network of vessels. Others believe that choroidal thickening (pachychoroid) in PCV suggests a different etiology from age related macular degeneration (AMD) and believe it to be part of the pachychoroid spectrum. ${ }^{2}$ Classification of $\mathrm{PCV}$ depends on polyp location, and can be subfoveal, juxtafoveal, extrafoveal, peripapillary, or peripheral. It

Correspondence: Sana Nadeem

Department of Ophthalmology, Foundation University Medical College \& Fauji Foundation Hospital, Rawalpindi

Email: sana.nadeem018@gmail.com

Received: December 8, 2019

Accepted: June 20, 2020 can also be classified into quiescent, exudative, or haemorrhagic, depending upon presentation. ${ }^{3}$ Dilated and multi-layered choroidal venules are involved in PCV; occlusion leads to ischemia and choroidal stasis, predisposing to serous PEDs and sub-retinal pigment epithelium (RPE) neovascularization, subsequently leading to sub-RPE or sub-retinal haemorrhages. The grape-like polyps are seen as a result of dilated venules and capillaries within the sub-RPE neovascular membrane. Definitive diagnosis can be made with indocyanine green angiography (ICGA), which delineates the polyps, but fluorescein angiography (FA) and optical coherence tomography (OCT) are also useful in its diagnosis. Ideal therapy for PCV is still unclear. Combination of intravitreal anti-VEGF and PDT is superior to either agent alone.,

\section{CASE PRESENTATION}

A 76-year-old, hypertensive lady presented to us in the out-patient department of Fauji Foundation Hospital, Rawalpindi. It is a tertiary care teaching hospital, affiliated with Foundation University Medical College. The patient complained of gradual, progressive visual loss of her right eye for the previous 3 years. She had undergone uneventful bilateral cataract surgeries 4 
years back. On examination, best corrected visual acuity (BCVA) was 6/60 in her right eye and 6/9 in her left with the Snellen chart. Fundus examination of the right eye revealed a large, bullous, serous PED involving the fovea, 3-4 DD (disc diameter) in size with an associated choroidal neovascular membrane $(\mathrm{CNVM})$ in the papillomacular bundle. CNVM was 1 DD in size, with surrounding multiple, serous PEDs, and scattered clumps of hard exudates and drusen. Orange aneurysmal dilatations could be seen on closer inspection. The left fundus showed multiple drusen, CNVM and multiple small PEDs in the inferior macular region. (Figure 1A and 1B). A diagnosis of Polypoidal choroidal vasculopathy (PCV) was made. She was taking Losartan (K) $50 \mathrm{mg}$ OD for hypertension.
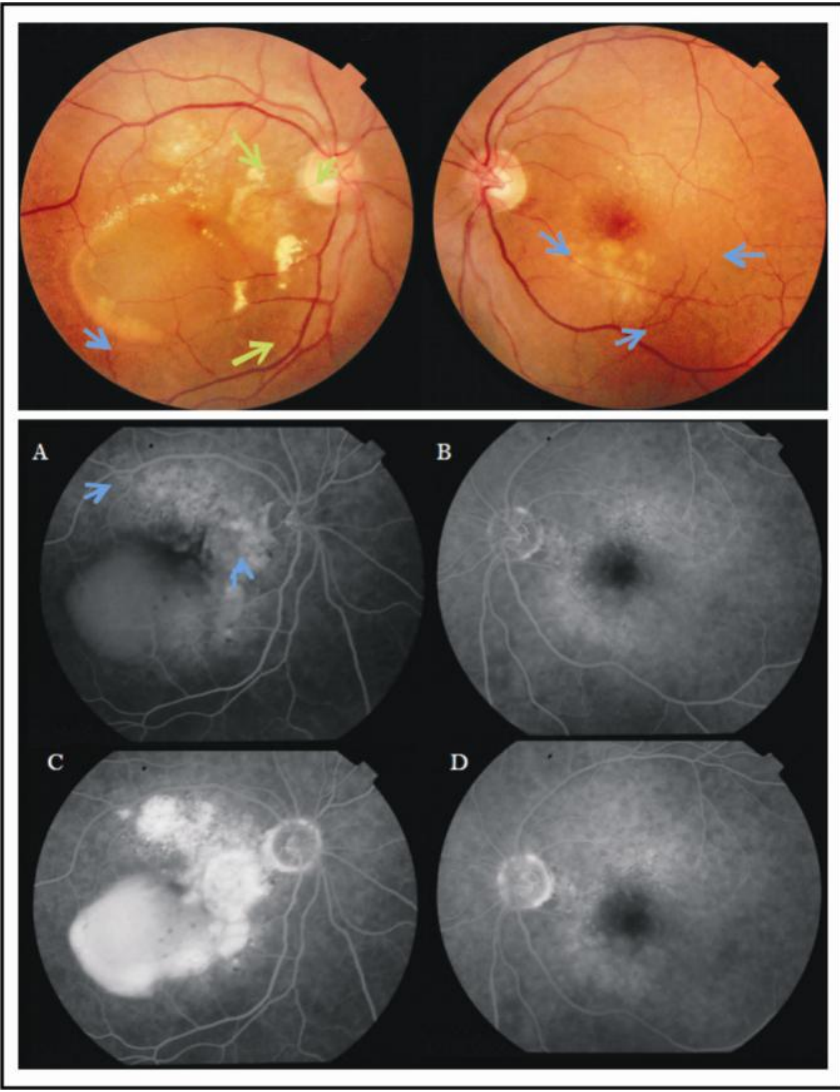

Fig. 1: Fundus photographs showing bilateral, multifocal, serous PEDs (blue arrows), CNVM, hard exudates and drusen. Orange aneurysmal dilatations (green arrows) can be seen on the right side only. A: FFA in mid-venous phase showing occult CNV adjacent to the disc and filling of the large PED B: Filling of multifocal PEDs and mottled hyperfluorescence C. Late frames showing CNVM, PED and diffuse macular leakage $D$. Late frames showing the small PEDs.
Cup-to-disc ratio (CDR) was 0.3 OD and 0.5 OS. Intraocular pressures were $25 \mathrm{~mm} \mathrm{Hg}$ OD and $22 \mathrm{~mm}$ $\mathrm{Hg}$ OS. Glaucoma was confirmed with OCT of the optic nerve and retinal nerve fiber layer. She was started on Timolol eye drops $0.5 \%$ BD and Ketorolac tromethamine eye drops $0.4 \%$ TDS. FFA showed occult CNVM OD (Figure 1). OCT of the macular region showed a large, bullous PED OD, cystic degeneration of the overlying retina, with multifocal PEDs and CNVM. On the left side, multifocal, small PEDs were evident [Figure 2]. Indocyanine green angiography (ICGA) was not available in our city, so the aneurysms could not be highlighted. We decided to treat her right eye with intravitreal Bevacizumab injections $1.25 \mathrm{mg}$ in $0.05 \mathrm{ml}$. The left eye was kept on observation due to good vision. Six intravitreal injections of Bevacizumab were administered on the right side.

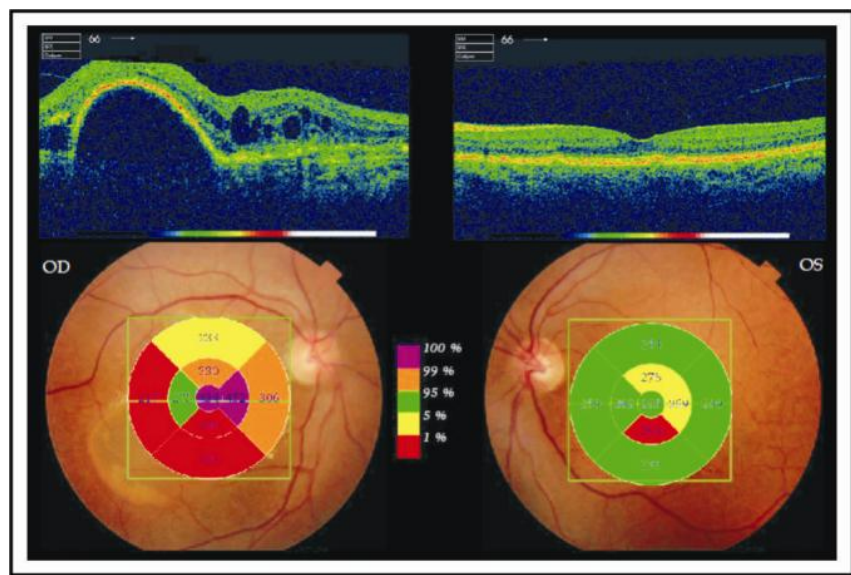

Fig. 2: OCT showing the macular thickness maps and evident PEDs.

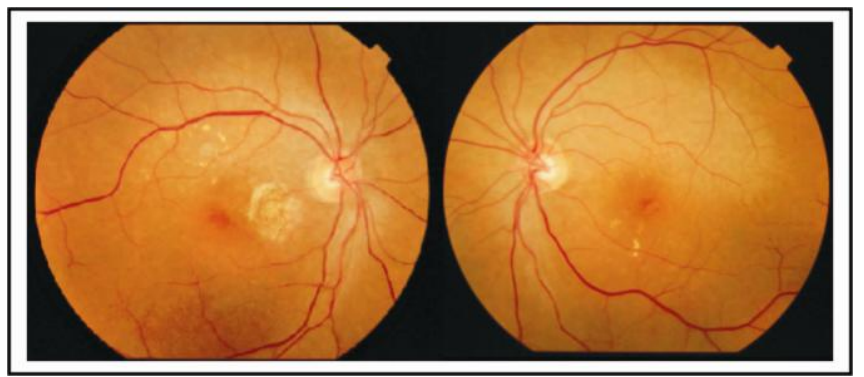

Fig. 3A: Fundus photographs at 1.5 year of follow up showing scarring from the CNVM OD with resolved PEDs OU.

Fig. 3B: Red-free photographs showing the improvement.

At 19 months of follow up, her visual acuity in right eye had improved to 6/36, PEDs had resolved 
and macular thickness profile was improved. The left eye also showed improvement but visual acuity remained stable at 6/9 [Figure 4A \& 4B]. OCT Macula revealed reduced size of the PEDs on both sides, with mild cystic retinal spaces OS [Figure 4].

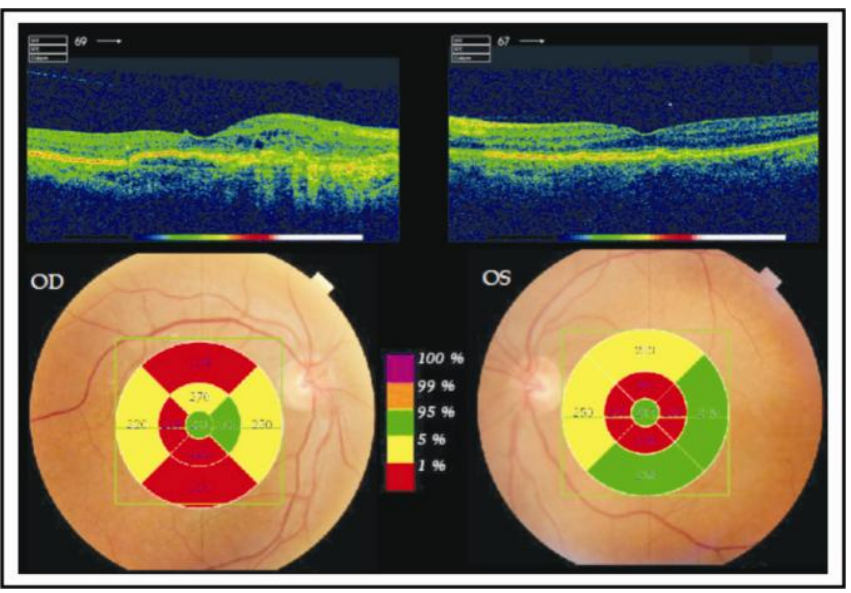

Fig. 4: OCT Macula showing resolution of the PEDs bilaterally.

\section{DISCUSSION}

Age related macular degeneration is the third leading cause of blindness in East Asia. ${ }^{6} \mathrm{PCV}$ has a higher prevalence amongst Asians (22.3\% to 61.6\%) as compared to Caucasians. Shared risk factors for AMD and PCV are smoking, raised C-reactive protein, plasma homocysteine and genetic factors. ${ }^{6,7}$

PCV is widely believed to be a type of exudative AMD or choroidal neovascularization, although others argue it to be a distinct choroidal vasculature abnormality. Features distinguishing PCV from typical AMD are: its distinct polypoidal lesions, often extramacular location, natural course and treatment response. $^{8}$

Diagnosis of PCV can be made clinically and confirmed by investigations. FFA usually shows occult $\mathrm{CNV}$, although rarely polyps can be visualised. ICG is the investigation of choice for delineating the diagnostic polyps. OCT shows characteristic domeshaped RPE elevations, which are highly reflective with moderately reflective polypoidal activity within the PEDs. The double-layer sign is composed of the RPE and the inner boundary of the Bruch membrane/ choriocapillaris complex. Fundus autofluorescence (FAF) and OCTA help us in the diagnosis and management of PCV.,
Natural history of PCV is variable and reveals a favourable course in $50 \%$ cases, with spontaneous regression of polyps. Visual morbidity can occur with polyp rupture, subsequent sub-retinal and vitreous haemorrhage, RPE and photoreceptor degeneration. ${ }^{10}$ The left eye in our patient showed spontaneous resolution of polyps and PEDs without treatment. Therapeutic approaches include thermal laser, PDT with Verteporfin, anti-VEGF agents and combined PDT/anti-VEGF therapy. Combination therapy is often considered to be the one yielding superior results. ${ }^{1-6,10}$ In our case, favourable outcome in the right eye was achieved with anti-VEGF injections of Bevacizumab only.

\section{Conflict of Interest}

Authors declared no conflict of interest.

\section{REFERENCES}

1. Mori F, Eguchi S. Polypoidal choroidal vasculopathy. From the viewpoint of an Asian ophthalmologist. [Editorial]. Br J Ophthalmol 2007; 91: 1104-1105.

2. Kumar A, Kumawat D, Sundar MD, Gagrani M, Gupta B, Roop P, et al. Polypoidal choroidal vasculopathy: a comprehensive clinical update. Ther Adv Ophthalmol. 2019; 11: 2515841419831152.

3. Cheung CM, Lai YY, Ruamviboonsuk P, Chen SJ, Chen Y, Freund KB, et al. Polypoidal Choroidal Vasculopathy: Definition, Pathogenesis, Diagnosis, and Management. Ophthalmology, 2018; 125 (5): 708-724.

4. Tso MO, Suarez MJ, Eberhart CG. Pathologic study of early manifestations of polypoidal choroidal vasculopathy and pathogenesis of choroidal neovascularization. Am J Ophthalmol Case Rep. 2017; 4 (11): 176-180.

5. Kim JB, Nirwan RS, Kuriyan AE. Polypoidal Choroidal Vasculopathy. Curr Ophthalmol Rep. 2017; 5 (2): 176-186.

6. Weng HY, Huang TL, Chang PY, Wang JK. Oneyear outcome of combination therapy with intravitreal aflibercept and photodynamic therapy for polypoidal choroidal vasculopathy. BMC Pharmacol Toxicol. 2019; 20:29.

7. Wong CW, Wong TY, Cheung CM. Polypoidal Choroidal Vasculopathy in Asians. J Clin Med. 2015; 4 (5): 782-821.

8. Honda S, Matsumiya W, Negi A. Polypoidal Choroidal Vasculopathy: Clinical Features and Genetic Predisposition. Ophthalmologica. 2014; 231 (2): 59-74. 
9. Tan CS, Ngo WK, Lim LW, Tan NW, Lim TH. EVEREST study report 4: Fluorescein angiography features predictive of polypoidal choroidal vasculopathy. Clin Exp Ophthalmol. 2019; 47 (5): 614620.

10. Wong RL, Lai TY. Polypoidal choroidal vasculopathy: an update on therapeutic approaches. J Ophthalmic Vis Res. 2013; 8 (4): 359-371.

\section{Author's Designation and Contribution} Sana Nadeem; Assistant Professor: Principle author, Drafting of manuscript, Diagnosis, Assessment, Evaluation \& Follow up of Patient. 